Check for updates

Cite this: RSC Adv., 2017, 7, 45287

Received 25th July 2017

Accepted 11th September 2017

DOI: $10.1039 / \mathrm{c} 7 \mathrm{ra0} 0184 \mathrm{~g}$

rsc.li/rsc-advances

\section{Bionic anti-adhesive electrode coupled with maize leaf microstructures and $\mathrm{TiO}_{2}$ coating $\dagger$}

\author{
Zhiwu Han, (D) ${ }^{a}$ Jia Fu, $^{a}$ Xiaoming Feng, ${ }^{a}$ Shichao Niu, (D) *ab Junqiu Zhang ${ }^{a}$ \\ and Luquan Ren ${ }^{a}$
}

Recently, the electrosurgical electrode used in minimally invasive surgery is becoming more and more popular as it causes only a little trauma and slight pain, and has other advantages. However, its high working temperature, above $400{ }^{\circ} \mathrm{C}$, often results in serious tissue adhesion on the surface of the electrode, which seriously affects the normal operation of the surgery. In this paper, we develop a novel type of electrode with a coupled bionic anti-adhesive surface by fabricating microstructures inspired by the maize leaf and coating a thin layer of $\mathrm{TiO}_{2}$ film on it. The grid-like microstructures inspired by the maize leaf were fabricated by laser marking technology. Then, the sol-gel method was chosen to prepare the $\mathrm{TiO}_{2}$ coating. The surface characterization of the electrode substrate, bionic electrode and coupled bionic electrode was carried out after fabrication. Afterwards, the wettability and components of these were measured and analysed, respectively. Furthermore, in order to determine the adhesion behaviour of different electrodes, electric cutting experiments were performed on fresh isolated animal liver tissue in detail. Finally, the relationships between adhesion mass, cutting time and cutting depth were also measured. The results show that the coupled bionic electrode surface has the most effective anti-adhesion performance compared with the bare original electrode surface under a high temperature. The investigations carried out in this work offer a promising way to design and fabricate anti-adhesive surfaces working at higher temperatures.

\section{Introduction}

Compared with the traditional scalpel, the minimally invasive electrosurgical electrode is becoming more and more popular as it causes only a little trauma and slight pain, and there is a rapid recovery for patients. However, tissue adhesion to the surface of the electrode, due to its working temperature of above $400^{\circ} \mathrm{C}$, often triggers a lot of detrimental clinical events, such as high temperature burns, carbonized eschar, biofouling and so on., ${ }^{1,2}$ Currently, there are two main ways to reduce tissue adhesion: chemical approaches and physical tactics. ${ }^{1,3}$ The chemical approach mainly refers to coating a thin-layer film of both of organic polymers and inorganic polymers, which are anti-adhesive, such as silicon, ceramics, Teflon, and polyethylene oxide. ${ }^{4-8}$ These various coatings are aimed at preventing protein adhesion or reducing van der Waals forces. ${ }^{9}$ However, these coatings are often extremely unstable and volatilize some toxic substances during surgery. The physical tactic, often referring surface modifications of the electrode by

${ }^{a}$ Key Laboratory of Bionic Engineering (Ministry of Education), Jilin University, Changchun 130022, China. E-mail: niushichao@jlu.edu.cn

${ }^{b}$ State Key Laboratory of Automotive Simulation and Control, Jilin University, Changchun 130022, China

$\dagger$ Electronic supplementary information (ESI) available. See DOI: $10.1039 / \mathrm{c} 7 \mathrm{ra} 08184 \mathrm{~g}$ processing some micro/nano-structures on the electrode surface, is aimed at reducing the actual contact area between the organism tissue and the electrode. ${ }^{10}$ However, these micro/ nano-structures can be easily damaged so that the modified electrode fails to work effectively.

To solve the problems described above, the principle of coupled bionics was adopted to fabricate a type of effectively anti-adhesive electrode via the combination of a bionic microstructure and chemical coatings. During long-term evolution and selection, living organisms have ingeniously evolved functional surface structures and heterogeneities to create highperformance biological materials from a fairly limited choice of elements and compounds. The translation of such design motifs into synthetic materials offers a spectrum of feasible pathways towards unprecedented properties and functionalities that are favourable for practical uses in a variety of engineering and medical fields. After billions of years of evolution, various species have coped with 'survival of the fittest' ${ }^{11,12}$ and nature has provided a lot of inspiration for engineers and scientists. ${ }^{13-15}$ Recently, anti-adhesion properties have become one of the most important hot spots among many excellent features of the organisms. ${ }^{16-20}$ The biological community provides us with a lot of anti-adhesion models, such as the lotus leaf, ${ }^{21,22}$ Nepenthes species, ${ }^{23,24}$ gecko spines ${ }^{25}$ some insect wing cuticles $^{26}$ and so on. However, few studies have taken higher 
temperature conditions into account in the anti-adhesive performance. In our previous study, we found that maize leaves have a lower adhesive property, and the anti-adhesive property was enhanced with an increase of temperature due to the ambient relative humidity $(\mathrm{RH})$ changes and the nonsmooth ridge tops on the surface of it. ${ }^{27}$

Compared with other kinds of coatings, $\mathrm{TiO}_{2}$ coatings have been widely used in recent years owing to their stability, nontoxicity and superhydrophilicity, which leads to $\mathrm{TiO}_{2}$ coatings with a good self-cleaning performance. ${ }^{16,28}$ In this work, inspired by the excellent anti-adhesive property of the maize leaf and taking the outstanding self-cleaning of $\mathrm{TiO}_{2}$ coating into account, a kind of coupled bionic electrode was fabricated. Laser marking was used to fabricate the maize leaf-inspired microstructure on the surface of the coupled bionic electrode because laser marking is powerful and it is easy to obtain surface microstructures with it. ${ }^{29-32}$ Furthermore, the sol-gel method was chosen to prepare the $\mathrm{TiO}_{2}$ coating. ${ }^{33,34}$ To understand more about the adhesion behaviour of the coupled bionic electrode, an electric cutting experiment was further performed on fresh isolated animal liver tissue. The relationships between the adhesion mass, cutting time and cutting depth were investigated in detail. The results show that the coupled bionic electrode surface anti-adhesive property behaves well compared with the bare original electrode surface.

\section{Results and discussion}

The surface morphology and changes in adhesion force related to the temperature of the maize leaf are shown in Fig. 1. Fig. 1(a) shows the macroscopic appearance of a maize leaf. A typical scanning electron microscope (SEM) image of the surface of

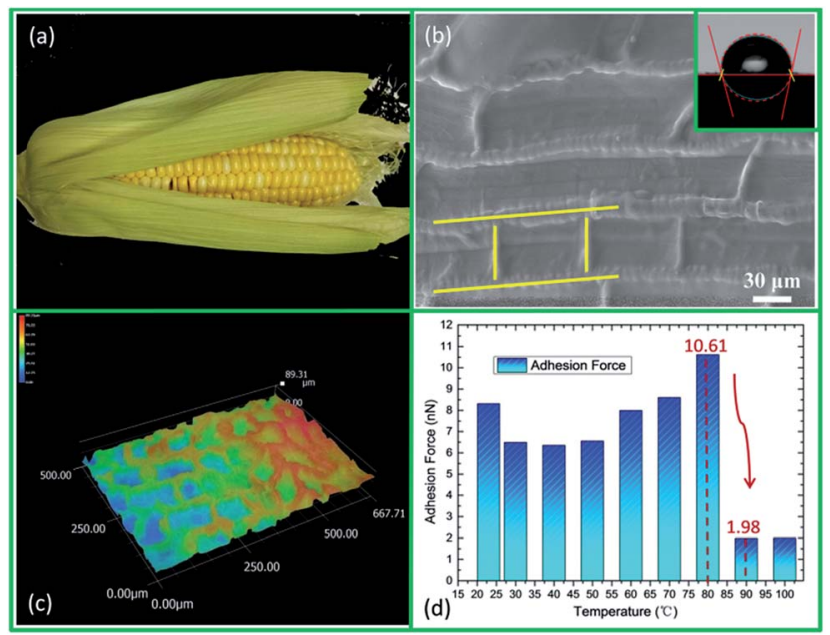

Fig. 1 (a) Digital image of a set of maize leaves with a corncob. (b) The SEM image of a maize leaf surface with a static CA image, showing the grid-like structure and the hydrophobicity of the maize leaf. (c) The SDFM image of a maize leaf, intuitively showing that there are plenty of small air pockets on the maize leaf surface. (d) The changes in adhesion force related to temperature. The adhesion force drops dramatically in the temperature range between $80-90{ }^{\circ} \mathrm{C}$, which indicates that the maize leaf has anti-adhesion properties at high temperatures. a maize leaf is shown in Fig. 1(b). It is found that, in contrast to a kind of hierarchical structure, the maize leaf shows a single type of staggered grid-like structure. The grid-like structure is composed of transverse and longitudinal ridges. The widths of the ridges in two directions are about 6-10 $\mu \mathrm{m}$ and $2-5 \mu \mathrm{m}$, respectively. The distance between ridges in the two directions, however, is non-quantitative. The distance between the transverse ridges is about $20-40 \mu \mathrm{m}$. In the vertical direction, the ridges distribute randomly, and have no regularity. Therefore, the distance between the longitudinal ridges is nonquantitative. As characterized by super depth of field microscopy (SDFM), the transverse and longitudinal ridges interlace with each other, forming plenty of small air pockets, which reduce the actual contact area between the object and the maize leaf surface (Fig. 1(c)). Intuitively, the surface wettability of the maize leaf was characterized by the static contact angle (CA) (Fig. 1(b)). It is clearly seen that, due to its unique structure, the static CA of the maize leaf is about $104^{\circ}$, which indicates that the maize leaf is slightly hydrophobic $\left(\mathrm{CA}>90^{\circ}\right)$ instead of being superhydrophobic $\left(\mathrm{CA}>150^{\circ}\right)$. The changes in adhesion related to temperature $\left(23-100{ }^{\circ} \mathrm{C}\right)$ of the maize leaf are shown in Fig. 1(d). A temperature lower than $80{ }^{\circ} \mathrm{C}$ doesn't affect the adhesion force seriously. However, in the temperature range between $80-90{ }^{\circ} \mathrm{C}$, the adhesion force exhibits a sharp decrease. The adhesion force drops from $10.61 \mathrm{nN}$ to $1.98 \mathrm{nN}$ dramatically within $10{ }^{\circ} \mathrm{C}$, and there is no upward trend from that point, which indicates that the maize leaf has the property of antiadhesion under high temperatures $\left(>80^{\circ} \mathrm{C}\right)$.

According to our study, the reduction of adhesion on the maize leaf surface at high temperatures may be explained by the ambient relative humidity ( $\mathrm{RH}$ ) changes and the non-smooth top of the ridges. ${ }^{27}$ Herein, the theory is not elaborated any further.

Inspired by the smart microstructure of the maize leaf surface, a set of bionic minimally invasive electrosurgical electrodes are designed with grid-like structures (Fig. S1 $\dagger$ ). Table 1 shows the structural parameters of 9 kinds of bionic electrodes. The height of the ridges in two directions is $80 \mu \mathrm{m}$ and has no change (Fig. S2(a) $\dagger$ ). The grid-like microstructure of the bionic electrode surface is shown in Fig. 4(d).

The whole fabrication process of the coupled bionic electrode is shown in Fig. 2. To confirm the cleanliness, all the

Table 1 The structural parameters of the bionic electrode ${ }^{a}$

\begin{tabular}{llll}
\hline Sample & $W(\mu \mathrm{m})$ & $L_{1}(\mu \mathrm{m})$ & $L_{2}(\mu \mathrm{m})$ \\
\hline 1 & 100 & 400 & 600 \\
2 & 100 & 500 & 800 \\
3 & 100 & 600 & 1000 \\
4 & 150 & 400 & 800 \\
5 & 150 & 500 & 1000 \\
6 & 150 & 600 & 600 \\
7 & 200 & 400 & 1000 \\
8 & 200 & 500 & 600 \\
9 & 200 & 600 & 800
\end{tabular}

${ }^{a} W$ : the width of both transverse and longitudinal ridges; $L_{1}$ : the distance between transverse ridges; $L_{2}$ : the distance between longitudinal ridges. 


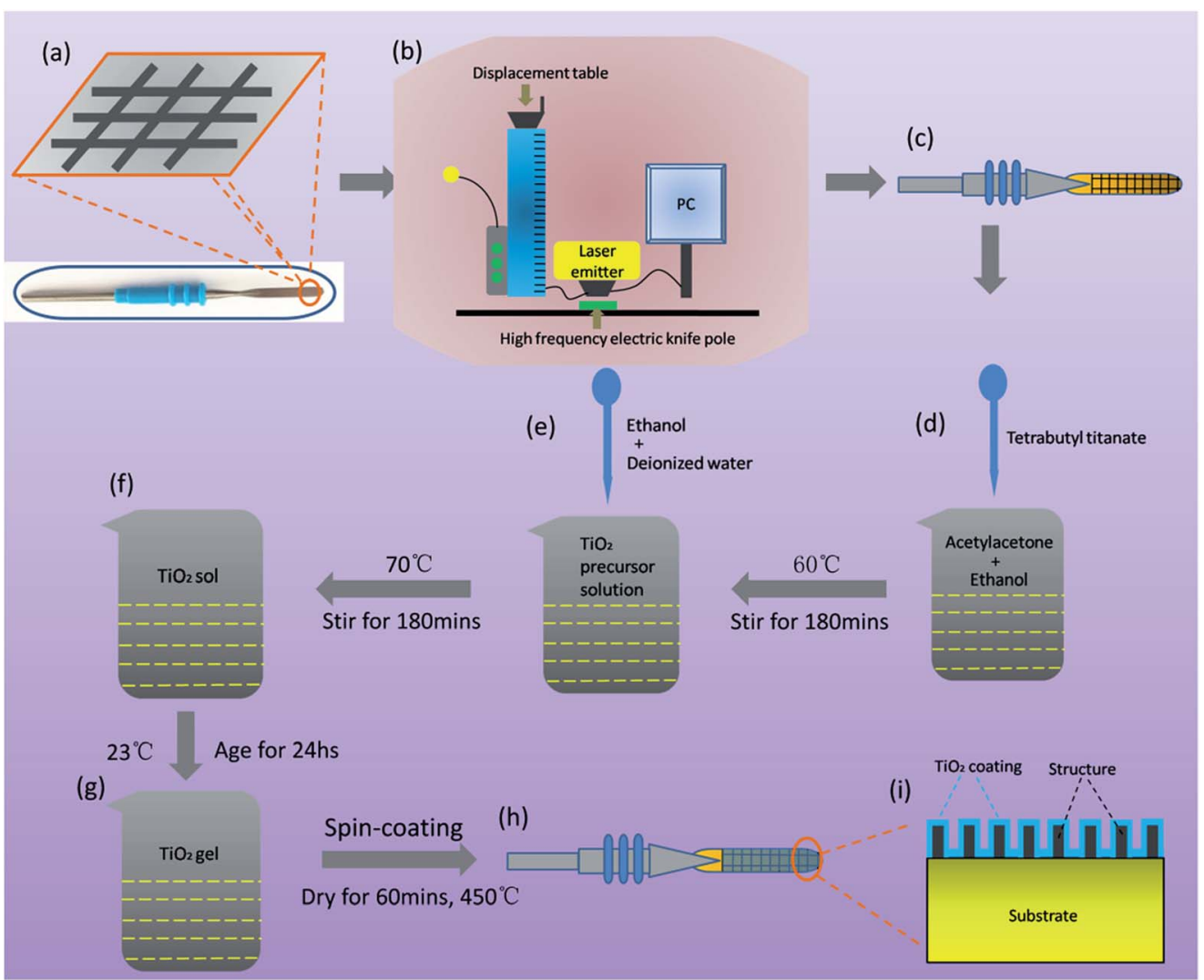

Fig. 2 Fabrication process of the coupled bionic electrode. (a)-(c) Fabrication process of the bionic electrode via laser marking. (d)-(h) The preparation of the $\mathrm{TiO}_{2}$ coating on the bionic electrode surface by the sol-gel method. (i) A diagram inside a coupled bionic electrode.

electrode substrates are soaked by ethanol, washed with ultrasonic for 20 minutes, and dried naturally in the air for 48 hours. After two-dimensional (2D) modelling of the bionic electrode with Autodesk Computer Aided Design (Auto CAD 2008) according the structural parameters in Table 1, the laser marking to fabricate the bionic electrode is chosen (Fig. 2(b) and (c)). Laser marking is a powerful tool to fabricate a variety of materials at the microscale. Using laser pulses to process the metal surface is an effective way to obtain surface microstructures. $^{29-32}$ Taking the material and characteristics of the electrode substrate into account, a set of machining parameters are determined and shown in Table 2.

Table 2 Processing parameters of the laser marking machine

\begin{tabular}{|c|c|c|c|}
\hline Index & Parameter & Index & Parameter \\
\hline Laser wavelength & $\begin{array}{l}\text { ND:YAG/ } \\
1064 \mathrm{~nm}\end{array}$ & $\begin{array}{l}\text { Laser marking } \\
\text { speed }\end{array}$ & $3 \mathrm{~mm} \mathrm{~s}^{-1}$ \\
\hline $\begin{array}{l}\text { Laser beam } \\
\text { frequency }\end{array}$ & $20 \mathrm{kHz}$ & Machining depth & $\leq 2 \mathrm{~mm}$ \\
\hline $\begin{array}{l}\text { Laser beam } \\
\text { quality }\end{array}$ & $M_{2}<1.3$ & Times of marking & 6 \\
\hline $\begin{array}{l}\text { Laser pulse } \\
\text { duration }\end{array}$ & $5 \mu \mathrm{m}$ & $\begin{array}{l}\text { Power } \\
\text { consumption }\end{array}$ & $\leq 0.6 \mathrm{KW}$ \\
\hline Current & $10 \mathrm{~A}$ & Power demand & $220 \mathrm{~V} \mathrm{AC} / 50 \mathrm{~Hz}$ \\
\hline Laser mode & $\mathrm{TEM}_{00}$ & Machining range & $\leq 1 \mathrm{~mm}$ \\
\hline
\end{tabular}

Before preparation of the $\mathrm{TiO}_{2}$ coating on the bionic electrode surface, 9 kinds of bionic electrodes are washed ultrasonically for 20 minutes and dried naturally in the air repeatedly. In order to ensure the uniformity of the $\mathrm{TiO}_{2}$ coating, the sol-gel method is chosen to prepare the $\mathrm{TiO}_{2}$ gel. Firstly, tetrabutyl titanate $(5 \mathrm{~mL})$ is added into the solution of acetylacetone $(5 \mathrm{~mL})$ and anhydrous ethanol $(75 \mathrm{~mL})$ at room temperature, and the mixed solution is stirred for $180 \mathrm{~min}$ at $60{ }^{\circ} \mathrm{C}$, which serves as the $\mathrm{TiO}_{2}$ precursor solution. In the second step, a mixed solution consisting of anhydrous ethanol $(15 \mathrm{~mL})$ and deionized water $(15 \mathrm{~mL})$ is slowly added to the $\mathrm{TiO}_{2}$ precursor solution prepared before, and the new mixed solution is stirred for $180 \mathrm{~min}$ at $75{ }^{\circ} \mathrm{C}$ and becomes a yellowish transparent $\mathrm{TiO}_{2}$ sol after stirring. After 36 hours of rest at room temperature, the yellowish transparent $\mathrm{TiO}_{2}$ sol eventually turns into a $\mathrm{TiO}_{2}$ gel, which is now ready to prepare the $\mathrm{TiO}_{2}$ coating on the bionic electrode surface. Considering the requirements of the coating and the size of the substrate, the $\mathrm{TiO}_{2}$ coating is prepared by a spin-coating method. It is worth noting that, in order to obtain uniformity of the $\mathrm{TiO}_{2}$ coating, a low gear (550 $\mathrm{rpm}$ ) of the coater is chosen at first and then a higher gear (2000 $\mathrm{rpm}$ ) is chosen. The time of the coating of the two gears is $10 \mathrm{~s}$. After spin-coating, the pre-coupled bionic electrode is dried in the drying oven for $60 \mathrm{~min}$ at $450{ }^{\circ} \mathrm{C}$. Then, the coupled bionic electrodes are fabricated successfully. The coating thickness is about 10-15 $\mu \mathrm{m}$ (Fig. 2(d)-(h)). A diagram of inside a coupled 
bionic electrode is shown in Fig. 2(i). It is clearly seen that the substrate surface is processed into a composite surface consisting of bionic microstructures with a thin layer of $\mathrm{TiO}_{2}$ coating around them. The single $\mathrm{TiO}_{2}$ coating on the electrode substrate is also prepared for later comparison.

The SEM images of the electrode substrate, bionic electrode and coupled bionic electrode at different magnifications are shown in Fig. 3. The surface of the electrode substrate is smooth at low magnification and has some tiny traces of manufacture (Fig. 3(a) and (b)). The static CA of the electrode substrate is $71^{\circ}$. The surface of the bionic electrode has been processed into a grid-like one (Fig. 3(c)). The image at a high magnification shows the groove-like microstructure with a lot of micro/nanoripple-like structures on it in the middle of the grid, which are left by laser marking (Fig. 3(d)). The static CA $\left(148^{\circ}\right)$ of the bionic electrode shows a strong hydrophobicity (almost reaching superhydrophobicity: $\mathrm{CA}>150^{\circ}$ ) due to the combined effect of the maize leaf structure-like microgrids, microgroove and micro/nano-ripple-like structures (Fig. 3(c)). Superficially, there is no obvious difference between the coupled bionic electrode and the bionic electrode, which exhibits microgrids and microgrooves with ripple-like micro/nano-structures on it (Fig. 3(e)). However, it can be seen clearly from the high magnification SEM image that there are a lot of subtle irregular nanoscale cracks on the surface of the coupled bionic electrode because of the drying of the $\mathrm{TiO}_{2}$ coating (Fig. 3(f)). The clearer cracks' image is shown in Fig. S2(b). $\dagger$ Owing to the superhydrophilicity of the $\mathrm{TiO}_{2}$ coating and the hierarchical structure, the static CA of the coupled bionic electrode is $105^{\circ}$, which is smaller than the CA of the bionic electrode (Fig. 3(e)). Furthermore, the X-ray diffraction pattern of the $\mathrm{TiO}_{2}$ coating on the surface of coupled bionic electrode is shown in Fig. S3, $\dagger$ with 9 diffraction peaks $\left(2 \theta=25.3^{\circ}, 37.8^{\circ}, 48.1^{\circ}, 54.0^{\circ}, 55.1^{\circ}\right.$, $62.7^{\circ}, 68.8^{\circ}, 70.3^{\circ}$ and $75^{\circ}$ ) corresponding to a typical anatase phase pattern, clearly observed (JCPDS no. 21-1272), which are indexed as the (101), (004), (200), (105), (221), (204), (116), (220) and (215) planes, respectively. In addition, the surface roughness (SRa) of electrode substrate is 1.344, and the coupled bionic electrode's is 3.610 (Fig. S4†).

Energy dispersive spectroscopy (EDS) spectra of the electrode substrate, bionic electrode and coupled bionic electrode are shown in Fig. 4. Before preparation of the $\mathrm{TiO}_{2}$ coating, the main elements of the electrode substrate and bionic electrode are $\mathrm{Fe}$ and $\mathrm{Cr}$, which are the essential elements of stainless steel. After laser marking, there are some quantitative changes but no qualitative change. The content of Fe increases from $59.96 \%$ to $63.61 \%$, and Cr's increases from $16.06 \%$ to $17.04 \%$ (Fig. 4(a) and (b)). After preparation of the $\mathrm{TiO}_{2}$ coating, however, the main elements of the coupled bionic electrode are $\mathrm{Ti}$ and $\mathrm{O}$, which reach up to $37.65 \%$ and $25.14 \%$, respectively. This further proves that the coating of $\mathrm{TiO}_{2}$ is obtained successfully via the sol-gel method on the surface of the bionic electrode (Fig. 4(c)). Fig. 4(d) shows the grid-like microstructure diagram of a bionic electrode surface.

In order to determine the adhesion behaviour of the bionic electrode and coupled bionic electrode, electric cutting experiments are performed (Fig. S5 $\dagger$ ). The fresh isolated animal liver tissue is cut by the equipment of a minimally invasive electrosurgical electrode with the electrode substrate, the bionic electrode, the single $\mathrm{TiO}_{2}$ coating surface and the coupled bionic electrode. The results are shown in Fig. 5. It is clearly seen that the surfaces of the electrode substrate, bionic electrode, single $\mathrm{TiO}_{2}$ coating surface and coupled bionic electrode are clean and neat before testing (Fig. 5(a), (c), (e) and (g)). However, liver tissue is adhering to the surface of the electrodes, more or less, after the electric cutting experiment. It is found that the tissue adhesions to the electrode substrate surface and the single $\mathrm{TiO}_{2}$ coating surface are most serious of all. The eschar tissue is

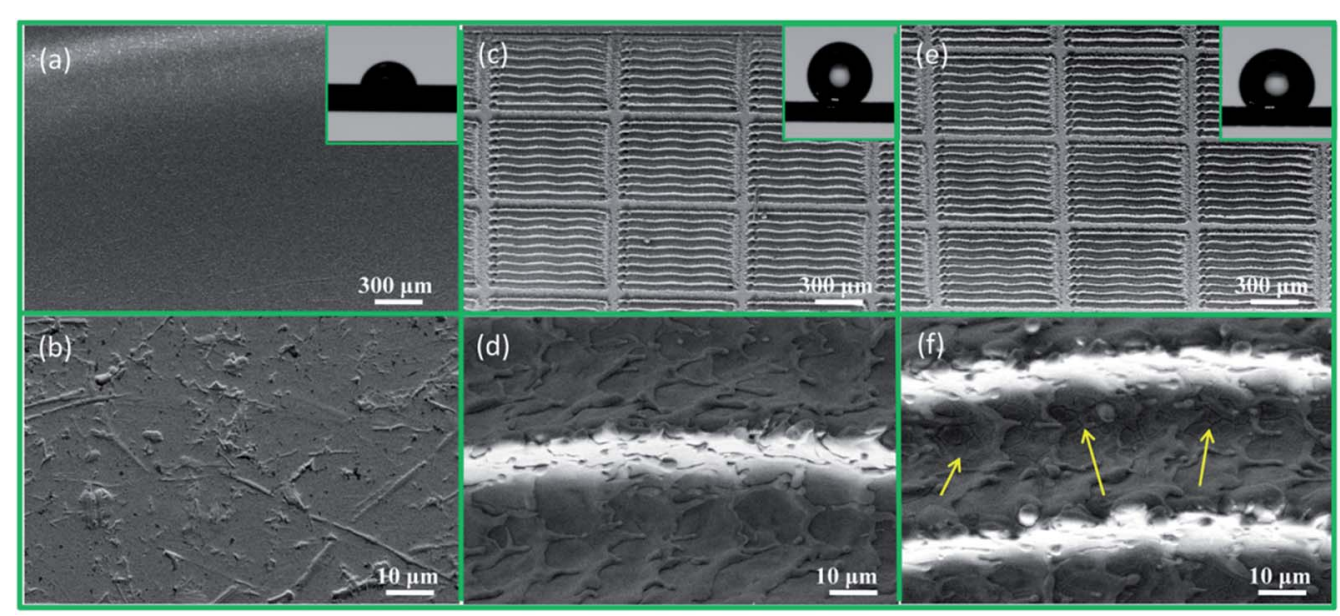

Fig. 3 SEM images of the electrode substrate, bionic electrode and coupled bionic electrode at different magnifications. (a, b) SEM images show that the surface of the electrode substrate is relatively smooth. (c) The low magnification SEM image shows that the surface of the bionic electrode is processed into a grid-like surface. (d) The high magnification SEM of the bionic electrode shows the microgroove with micro/nanoripple-like structures on it left by laser marking in the middle of a grid. (e) Low magnification SEM image of a coupled bionic electrode, showing that there is no big difference between the coupled bionic electrode and the bionic electrode. But the high magnification SEM image shows there are a lot of subtle cracks on the surface of coupled bionic electrode (f). 

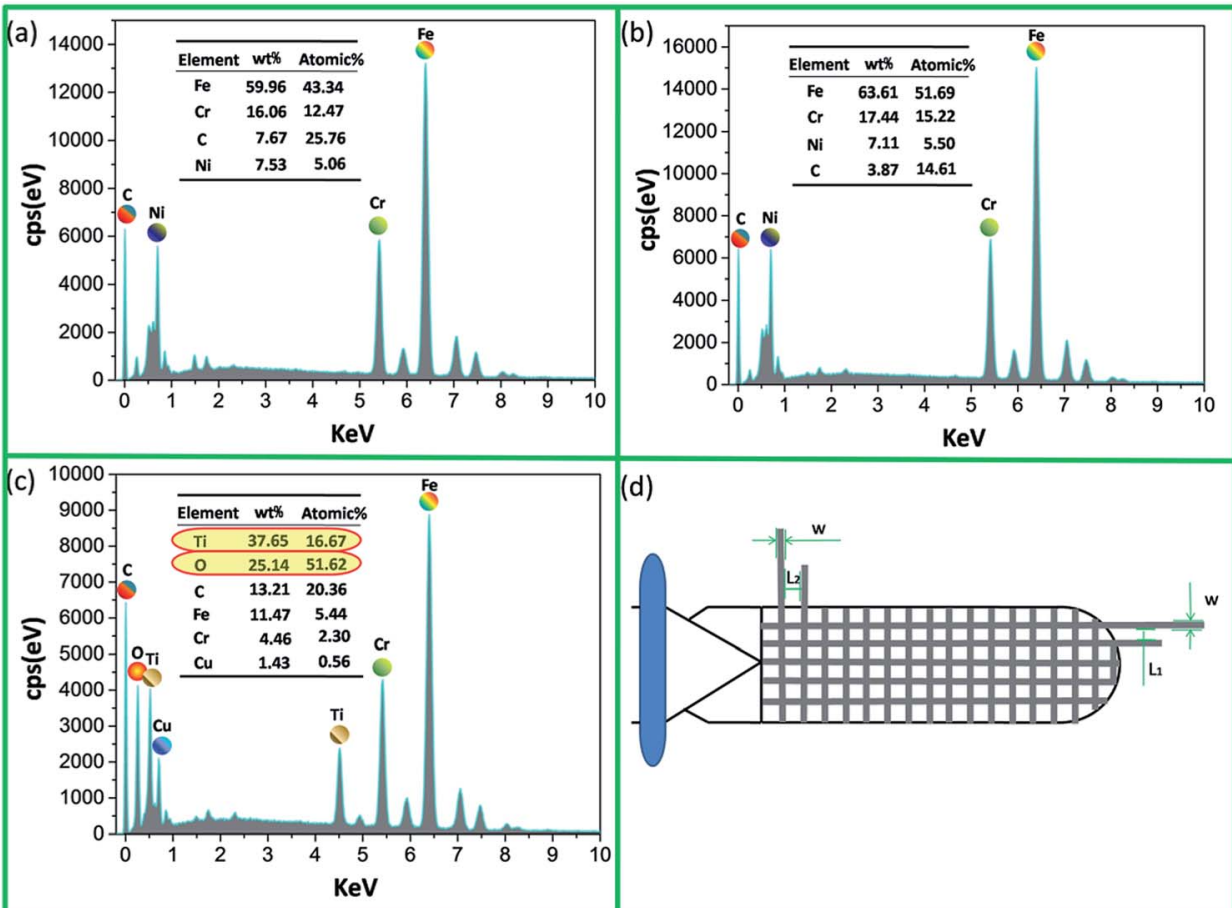

Fig. 4 (a) EDS spectrum of the electrode substrate. (b) EDS spectrum of the bionic electrode. (c) EDS spectrum of the coupled bionic electrode. These results indicate that the main elements of the coupled bionic electrode are $\mathrm{Ti}$ and $\mathrm{O}$, which further proves that the coating of $\mathrm{TiO}_{2}$ is obtained successfully. (d) The grid-like microstructure diagram of a bionic electrode surface.

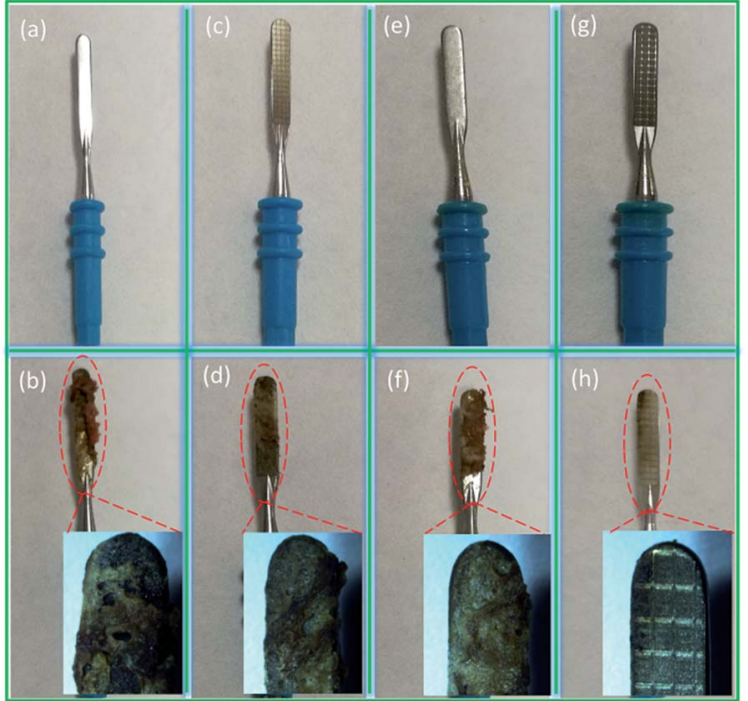

Fig. 5 Digital images of 4 kinds of electrodes before and after the electric cutting experiment. (a), (c), (e), (g) The digital images before cutting show the original clean surfaces of the electrode substrate, bionic electrode, single $\mathrm{TiO}_{2}$ coating on the electrode substrate and on the coupled bionic electrode, respectively. (b), (d), (f), (h) The adhesion behaviour of the electrode substrate, bionic electrode, single $\mathrm{TiO}_{2}$ coating on the electrode substrate and on the coupled bionic electrode, respectively, which reveal that the tissue adhesions to the electrode substrate surface and single $\mathrm{TiO}_{2}$ coating surface are the most serious of all, and the adhesion to the coupled bionic electrode is the least serious. almost all over the two kinds of electrode surfaces (Fig. 5(b) and (f)). The bionic electrode surface also has a thin layer of eschar tissue adhesion (Fig. 5(d)). However, almost no eschar tissue is adhered to the surface of the coupled bionic electrode. The coupled bionic electrode surface is only slightly dark, which indicates the least adhesion to the surface (Fig. 5(h)).

In order to further determine the adhesion property of the coupled bionic electrode, the relationship between adhesion mass and time is investigated in detail, as shown in Fig. 6(a). The whole experiment lasts $4 \mathrm{~s}$, and each time interval is $0.5 \mathrm{~s}$, which is calculated precisely by an electronic timer. In general, the adhesion mass of both the electrode substrate and the coupled bionic electrode show an upward trend as time goes on.

For the electrode substrate, the mass of adhesion has risen from 0 to $61 \mathrm{mg}$. The adhesion mass reaches a peak when it comes to $2.5 \mathrm{~s}$ and has no change from that point. In the interval from $1 \mathrm{~s}$ to $1.5 \mathrm{~s}$, the increment of the adhesion mass is the maximum, which is $26.1 \mathrm{mg}$. Compared with the electrode substrate, the coupled bionic electrode is much better. The mass of adhesion to the coupled bionic electrode surface has risen from 0 to $18 \mathrm{mg}$ within $3 \mathrm{~s}$ and has no change after that, which indicates that the coupled bionic electrode not only has a better anti-adhesion property, but also a slower adhesion velocity compared with that of the electrode substrate. Furthermore, the relationship between the adhesion mass and the cutting depth is also investigated, as shown in Fig. 6(b). The semi-circular end of the electrode is defined as the depth of $0 \mathrm{~mm}$. The interval is $2 \mathrm{~mm}$, and the total depth is $14 \mathrm{~mm}$. It is clearly seen that both the electrode substrate and the coupled 

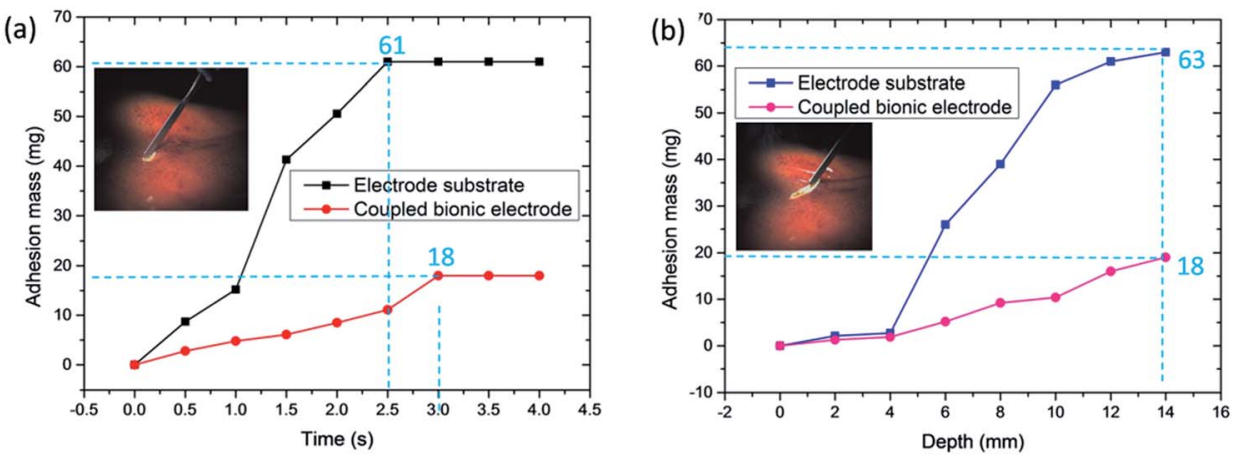

Fig. 6 (a) The contrastive line graphs of tissue adhesion mass related to electric cutting time between the electrode substrate and the coupled bionic electrode. (b) The contrastive line graphs of tissue adhesion mass related to the electric cutting depth between the electrode substrate and the coupled bionic electrode.

bionic electrode exhibit an overall ascending tendency and reach a peak at the end of the depth. The maximum adhesion mass to the surface of the electrode substrate is up to $63 \mathrm{mg}$. However, for the coupled bionic electrode it is only $18 \mathrm{mg}$, which is similar to the results of the relationship between adhesion mass and time. Both tests on the adhesion mass reveal that the coupled bionic electrode does have a better antiadhesive property for tissue.

\section{Conclusions}

In summary, inspired by the excellent anti-adhesive property of the maize leaf at a high temperature, and taking the outstanding superhydrophilic self-cleaning effect of a $\mathrm{TiO}_{2}$ coating into account, a new type of coupled bionic anti-adhesive electrode was fabricated. The coupled bionic anti-adhesive electrode surface consists of smart grid-like microstructures with a thin layer of $\mathrm{TiO}_{2}$ coating around them. After 2D modelling of the grid-like microstructures based on the maize leaf, laser marking was used to fabricate the bionic electrode. Then, the $\mathrm{TiO}_{2}$ coating was prepared by the sol-gel method. Furthermore, electric cutting experiments on fresh isolated animal liver tissue were performed in order to determine the adhesion behaviour of the coupled bionic anti-adhesive electrode. The results show that the single bionic anti-adhesive electrode exhibits a slightly better antiadhesive property with liver tissue. Compared with the bare original electrode surface, however, the coupled bionic antiadhesive electrode has the least adhesion to its surface, which indicates that the coupled bionic anti-adhesive electrode we fabricated shows an effective anti-adhesive property. Therefore, we anticipate that the coupled bionic effect of the microstructures of the maize leaf and $\mathrm{TiO}_{2}$ coating could inspire more researchers and scientists to design more robust engineering products, especially at a high temperature, in the future.

\section{Experimental section}

\subsection{Materials and chemical reagents}

Maize leaves were collected from the Chinese Academy of Agricultural Sciences. The scientific type of the maize is Zheng Dan 958 (validated in 2000, Henan Academy of Agricultural
Sciences, China). The flat headed minimally invasive electrosurgical electrode substrates, made of 304 medical stainless steel, were purchased from Kindly Electronic CO., Ltd. The fresh isolated animal liver tissue was obtained from the supermarket of Wal-Mart. All the chemical reagents used in the fabrication of the coupled bionic electrode were gained from the Changchun Institute of Applied Chemistry Chinese Academy of Sciences.

\subsection{Fabrication of the coupled bionic electrode}

After two-dimensional (2D) modelling of the bionic electrode, a laser marking machine (LSM: FB20-1) was used to fabricate the microstructures on the surface of the electrode substrate. With the $\mathrm{TiO}_{2}$ coating prepared, a magnetic heating mixer (MAM: 78-1) was used to mix the solution evenly. A spin coater (SP: Smart Coater-100) and tube furnace (TF: TL-1200) were chosen to coat the $\mathrm{TiO}_{2}$ coating and dry it, respectively.

\subsection{Measurements}

The multiscale 2D morphologies and structures of the maize leaves and all the electrodes were inspected on a scanning electron microscope (SEM: EOV-18). In addition, a super depth of field microscope (KEYENCE: VHX-6000) was also used to investigate the morphologies of the maize leaf.

Atomic force microscopy (AFM: Bruker's Dimension Icon Scan Asyst) was chosen to determine the adhesion force related to the temperature between the flat tip and the maize leaf surfaces, analysed by the software NanoScope Analysis.

A Confocal Laser Scanning Microscope (CLSM: LSM-800) was used to measure the surface roughness of the electrodes.

X-ray diffraction (XRD: Bruker's D8 Advance) was chosen to identify the phases formed on the surface of the coupled bionic electrode.

In order to investigate the wettability of the maize leaf surface and the electrodes, static contact angle (CA) measurements were carried out by a sessile droplet method (OCA20). The droplet volume is $5 \mu \mathrm{L}$.

Energy dispersive spectroscopy (EDS: X-Max ${ }^{\mathrm{N}} 150$ ) was used in order to investigate the elements and compositions of the electrode substrate, bionic electrode and coupled bionic electrode. 


\subsection{Electric cutting experiment}

The electric cutting experiment was accomplished by means of a set of minimally invasive electrosurgical electrodes (S900E), which was purchased from Kindy Electronic CO., Ltd. During the cutting experiment, the adhesion mass was weighed via an electronic analytical balance (EAB: FA-1204).

\section{Conflicts of interest}

There are no conflicts to declare.

\section{Acknowledgements}

This work was supported by the National Natural Science Foundation of China (No. 51325501, 51505183 and 51290292), China Postdoctoral Science Foundation Funded Project (No. 2015M571360), Joint Construction Project of Jilin University and Jilin Province (No. SF2017-3-4), and Outstanding Young Talent Fund of Jilin Province (No. 20170520095JH).

\section{Notes and references}

1 I. Sotiri, J. C. Overton, A. Waterhouse and C. Howell, Exp. Biol. Med., 2016, 241, 909-918.

2 G. Shepherd, P. Mohorn, K. Yacoub and D. W. May, Ann. Pharmacother., 2012, 46, 169-175.

3 K. D. Park, Y. S. Kim, D. K. Han, Y. H. Kim, E. H. B. Lee, H. Suh and K. S. Choi, Biomaterials, 1998, 19, 851-859.

4 D. C. Yates, P. Q. Shuster, K. V. Moran, D. A. Witt, J. R. Giordano and J. J. Kuhns, US Pat., 7780663, 2010.

5 J. H. Lee, S. K. Moon, K. M. Kim and K. N. Kim, Acta Odontol. Scand., 2013, 71, 168-174.

6 P. O. Davison, I. R. I. Mcnall, J. R. Tighe and A. B. Vankov, US Pat., 20130110108, 2013.

7 W. P. Heim and J. Brassell, US Pat., 2007087618 A2, 2007.

8 D. W. Greep, S. K. Horner and B. J. Walter, US Pat., 8439910, 2013.

9 S. Ebnesajjad, Fluoroplastics, vol. 1: non-melt processible fluoroplastics, Elsevier, USA, 2000.

10 C. W. Cheng, C. Y. Lin, W. P. Tseng, K. L. Ou and P. W. Peng, US Pat., 20130138103, 2013.

11 B. Bhushan, Philos. Trans. R. Soc., A, 2009, 367, 1445-1486. 12 S. Nishimoto and B. Bhushan, RSC Adv., 2012, 3, 671-690.

13 S. Yang, J. Ju, Y. He, X. Wang, S. Dou, K. Liu and L. Jiang, Small, 2014, 10, 294-299.
14 K. Liu and L. Jiang, Nano Today, 2011, 6, 155-175.

15 K. Liu and L. Jiang, ACS Nano, 2011, 5, 6786-6790.

16 Y. K. Lai, J. Y. Huang, Z. Q. Cui, M. Z. Ge, K. Q. Zhang, Z. Chen and L. F. Chi, Small, 2016, 12, 2203-2224.

17 G. S. Watson, D. W. Green, L. Schwarzkopf, X. Li, B. W. Cribb, S. Myhra and J. A. Watson, Acta Biomater., 2015, 21, 109-122.

18 E. V. Gorb, J. Purtov and S. N. Gorb, Sci. Rep., 2014, 4, 05154.

19 Y. Zhang, S. Qu, X. Cheng, X. Gao and X. Guo, Journal of Bionic Engineering, 2016, 13, 132-142.

20 J. L. Yong, Q. Yang, F. Chen, D. S. Zhang, G. Q. Du, H. Bian, J. H. Si and X. Hou, RSC Adv., 2014, 4, 8138-8143.

21 J. Wang, H. Chen, T. Sui, A. Li and D. Chen, Plant Sci., 2009, 175, 687-696.

22 L. Q. Chen, Z. Y. Xiao, P. C. H. Chan, Y. K. Lee and Z. G. Li, Appl. Surf. Sci., 2011, 257, 8857-8863.

23 H. W. Chen, P. F. Zhang, L. W. Zhang, H. L. Liu, Y. Jiang, D. Y. Zhang, Z. W. Han and L. Jiang, Nature, 2016, 532, 8589.

24 V. Bonhomme, H. Pellouxprayer, E. Jousselin, Y. Forterre, J. J. Labat and L. Gaume, New Phytol., 2011, 192, 545-554.

25 M. Spinner, S. N. Gorb and G. Westhoff, Proc. R. Soc. B, 2013, 280, 20132160.

$26 \mathrm{H}$. M. Hu, J. A. Watson, B. W. Cribb and G. S. Watson, Biofouling, 2011, 27, 1125-1137.

27 Z. W. Han, J. Fu, Y. Q. Fang, J. Q. Zhang, S. C. Niu and L. Q. Ren, Journal of Bionic Engineering, 2017, 14, 540-548.

28 H. Shi, Y. He, Y. Pan, H. H. Di, G. Y. Zeng, L. Zhang and C. L. Zhang, J. Membr. Sci., 2016, 506, 60-70.

29 J. Y. Long, P. X. Fan, D. W. Gong, D. F. Jiang, H. J. Zhang, L. Li and M. L. Zhong, ACS Appl. Mater. Interfaces, 2015, 7, 9858-9865.

30 S. Moradi, S. Kamal, P. Englezos and S. G. Hatzikiriakos, Nanotechnology, 2013, 24, 415302.

31 A. M. Kietzig, S. G. Hatzikiriakos and P. Englezos, Langmuir, 2009, 25, 4821-4827.

32 F. Chen, D. S. Zhang, Q. Yang, J. L. Yong, G. Q. Du, J. H. Si, F. Yun and X. Hou, ACS Appl. Mater. Interfaces, 2013, 5, 67776792.

33 A. Eshaghi, R. Mozaffarinia, M. Pakshir and A. Eshaghi, Ceramurgia Int., 2011, 37, 327-331.

34 T. Giannakopoulou, N. Todorova, T. Vaimakis, S. Ladas and C. Trapalis, J. Sol. Energy Eng., 2008, 130, 32-42. 